\title{
Suplementación vitamínica y mineral como estrategia para reducir la incidencia de cetosis bovina en el trópico bajo
}

\section{Vitamin and mineral supplementation as strategy to reduce the incidence of bovine ketosis under tropical conditions}

\author{
GARCÍA A. KATHERINE ${ }^{1 *}$ M.Sc, CAMPOS G. ROMULO ${ }^{1,2}$ Ph.D, \\ GIRALDO P. LEONIDAS ${ }^{1,2}$ MVZ.
}

\begin{abstract}
${ }^{1}$ Universidad Nacional de Colombia, Grupo de investigación Conservación, mejoramiento y utilización del ganado criollo Hartón del Valle y otros recursos genéticos animales en el suroccidente colombiano, sede Palmira.

universidad Nacional de Colombia, Facultad de ciencias agropecuarias, sede Palmira.
\end{abstract}

\section{Keywords:}

Cattle,

micronutrients, postpartum, lipid mobilization.

\section{Abstract}

The aim of this study was to evaluate the effect of mineral and vitamin supplementation on the incidence of ketosis under tropical conditions. 21 Holstein cows were divided into 3 groups: control T1, T2 iodine supplementation, T3 mixed mineral and vitamin supplementation. The experiment started 30 days before partum and culminated in the day 105 postpartum. Bi-weekly intramuscular administration of treatments performed, blood samples and urine samples were taken. $\beta$-hydroxybutirate (BHB), non esterified fatty acids (NEFA) and ketones were the metabolites analized. For statistical analysis, was done a repeated measures design, a descriptive analysis and comparison of means. No statistical differences were found between groups, but it did among periods, showing the highest values around calving. BHB, NEFA and ketones showed a higher concentration in the control group, while the lowest values were found in T3. Between $\mathrm{BHB}$ and ketones was found a high correlation $(r=0,72)$. Supplementation with vitamins and minerals allowed for better adaptation to transition period with a lower mobilization of body reserves. The correlation between ketones and $\mathrm{BHB}$ allows propose the detection of ketones in the urine as a effective test for the diagnosis of subclinical ketosis.

\section{Resumen}

El objetivo de este estudio fue evaluar el efecto de la suplementación mineral y vitamínica sobre la incidencia de cetosis en trópico bajo. 21 vacas Holstein fueron divididas en 3 grupos: T1 control, T2 suplementación yodada, T3 suplementación mineral-vitamínica mixta. El experimento inició 30 días antes del parto y culminó al día 105 posparto. Quincenalmente se realizó aplicación intramuscular de los tratamientos y se colecto sangre y orina. Los metabolitos analizados fueron beta-hidroxibutirato (BHB), ácidos grasos no esterificados (NEFA) y cetonas. Para el análisis estadístico se realizó diseño de medidas repetidas en el tiempo, análisis descriptivo y comparaciones entre medias. No se encontraron diferencias estadísticas entre grupos, pero si entre periodos, evidenciando valores altos alrededor del parto. BHB, NEFA y cetonas presentaron concentración mayor en T1, mientras en T3 se hallaron los menores valores. Entre BHB y cetonas se presentó una alta correlación $(r=0,72)$. La suplementación con vitaminas y minerales permitió una mejor adaptación al periodo de transición con menor movilización de reservas corporales. La correlación entre cetonas y BHB permite la detección de cetonas en orina como una prueba eficaz en diagnóstico de cetosis subclínica. 


\section{Introducción}

Durante el periodo de transición comprendido entre tres semanas antes y tres semanas después del parto, las vacas presentan diversos cambios fisiológicos que las hacen vulnerables a desarrollar balance energético negativo (BEN). Después del parto la producción de leche aumenta rápidamente mientras que el consumo de materia seca se encuentra limitado, esta situación ocasiona un desbalance entre los nutrientes requeridos y los nutrientes consumidos, dando lugar a la movilización de reservas corporales y una considerable pérdida de peso (GARRO et al., 2013).

La alta tasa de movilización de reservas conlleva al aumento en la concentración de ácidos grasos no esterificados (NEFA) en el plasma (GROSS et al., 2013). Los NEFA que llegan al hígado son convertidos en Acetil-CoA en la mitocondria del hepatocito por medio del proceso de $\beta$-oxidación. El acetil-CoA puede ser incorporado al ciclo de Krebs para la obtención de energía en forma de ATP; sin embargo, para esto es necesario el oxalacetato, el cual proviene de precursores glucogénicos como el propionato, acetato, glicerol o determinados aminoácidos. Durante el periodo de transición el oxalacetato es insuficiente por la falta de precursores glucogénicos, y por tanto el Acetil CoA no puede ingresar al ciclo de Krebs y sufre una oxidación incompleta formando los tres cuerpos cetónicos, acetona, aceto-acetato y beta-hidroxibutirato (DUQUE et al., 2011). La acumulación de cuerpos cetónicos puede llevar al animal a un cuadro de cetosis o también puede presentarse la re-esterificación de los NEFA a triglicéridos, los cuales se almacenan en los hepatocitos ocasionando la formación de hígado graso (WEBER et al., 2013).

La cetosis puede presentarse en forma clínica o subclínica. La cetosis clínica presenta signos tanto nerviosos como digestivos, e incluyen salivación, masticación, andar tambaleante, incoordinación motora, ceguera, caminar en círculos, déficit propioceptivo, presión de la cabeza contra objetos, ceguera aparente, pica, salivación, hiperestesia, vocalización constante, trémores moderados y tetania, heces duras, disminución en el consumo de alimento, anorexia y caída en la producción de leche (NORO y BARBOZA, 2012). La forma subclínica es la más frecuente, y se caracteriza por un incremento en los cuerpos cetónicos en sangre sin ningún síntoma clínico evidenciable, lo que hace su diagnóstico más complejo; se presenta disminución en la producción de leche y aumento en la susceptibilidad a otras enfermedades (BERGE y VERTENTEN, 2014).

La concentración de beta-hidroxibutirato (BHB) en sangre ha sido la prueba que mayor aceptación ha tenido en el diagnóstico de cetosis, caracterizándose por niveles de BHB en suero entre 1,00 y $1,40 \mathrm{mmol} / \mathrm{L}$ en la forma subclínica, y superiores a $1,4 \mathrm{mmol} / \mathrm{L}$ en la forma clínica (SUTHAR et al., 2013). Sin embargo, existen otras pruebas como la medición de BHB en leche (KROGH et al., 2011) y la determinación de cetonas en orina, mediante las cuales podría identificarse este desorden metabólico (CAMPOS et al., 2005).

La disminución en el consumo de alimento que se presenta durante el periodo de transición, afecta no solo el aporte de macronutrientes como proteína y energía al organismo, sino que también afecta el aporte de micronutrientes como lo son las vitaminas y los minerales, los cuales, a pesar de ser necesarios en pequeñas cantidades participan en diferentes procesos metabólicos esenciales para la sobrevivencia y la actividad productiva y reproductiva de las vacas lecheras. Los requerimientos de estos micronutrientes dependen del estado fisiológico y el nivel productivo de los animales, donde vacas de alta producción tendrán requerimientos mayores en el final de la gestación y el inicio de la lactancia, por la alta demanda de nutrientes que se presenta para crecimiento fetal y producción de leche (WILDE, 2006).

En el presente trabajo se realizó suplementación yodada y suplementación mineral mixta y vitamínica con el objetivo de evaluar su efecto sobre la incidencia de cetosis subclínica en vacas de producción lechera en condiciones de trópico bajo, además de analizar la correlación entre pruebas sanguíneas y de orina en el diagnóstico de este desorden metabólico. 


\section{Materiales y métodos}

La investigación se llevó a cabo en un sistema comercial semiintensivo de producción lechera, ubicado a $3^{\circ}, 57 \mathrm{~N}, 76^{\circ}, 15 \mathrm{~W}$; con altitud de $973 \mathrm{msnm}$, temperatura promedio de $25^{\circ} \mathrm{C}$, y precipitación media anual de $1000 \mathrm{~mm}$, encontrándose según la clasificación de Holdridge en una formación ecológica de Bosque Seco Tropical (Bs-T) (HOLDRIDGE, 1987).

El experimento contó con la licencia para experimentación animal otorgada por el comité de ética en investigación de la Universidad Nacional de Colombia, sede Palmira.

Los animales contaban con una composición genética basada en Holstein con producción

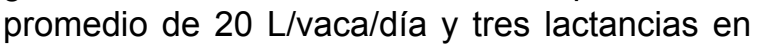
promedio. Las vacas se encontraban bajo un sistema de pastoreo rotacional con pasto estrella Cynodon plectostachyus, suplementado con concentrado artesanal a base de maíz, torta de soya y mogolla, la composición nutricional de estos alimentos se encuentra en la Tabla 1. El concentrado artesanal se suministró durante los ordeños, en una cantidad de $1 \mathrm{Kg}$ por cada 2.5 litros de leche, a partir de una base forrajera para 8 litros de leche.

Tabla 1. Análisis bromatológico del pasto estrella y el suplemento suministrado a los animales experimentales

\begin{tabular}{lcc}
\hline \multicolumn{1}{c}{ Nutriente (\%) } & $\begin{array}{c}\text { Pasto estrella } \\
\text { (Cynodon plectostachyus) }\end{array}$ & Suplemento \\
\hline Materia seca & 18,87 & 84,94 \\
Cenizas & 9,53 & 7,68 \\
Proteína & 18,06 & 27,56 \\
Extracto etéreo & 2,32 & 5,09 \\
Carbohidratos & 5,22 & 37,06 \\
FDN $^{*}$ & 64,87 & 22,61 \\
FDA $^{*}$ & 33,63 & 6,42 \\
LDA $^{*}$ & 4,18 & 1,18 \\
Hemicelulosa & 31,24 & 16,19 \\
Celulosa & 29,45 & 7,47 \\
NDT * & 55,6 & 71,9 \\
Energía digestible & 2,6 & 2,8 \\
(Mcal/Kg*MS) & & \\
\hline
\end{tabular}

* FDN: Fibra detergente neutra, FDA: Fibra detergente Acida, LDA: Lignina detergente acida, NDT: Nutrientes digestibles totales, los valores están expresados en base seca.
21 animales fueron seleccionados por mayor producción de leche y por encontrarse en un periodo cercano a parto, fueron distribuidos de forma aleatoria en 3 grupos experimentales ( $T 1$, T2 y T3) de 7 vacas cada uno.

Durante el experimento los grupos mantuvieron la alimentación base suministrada en la finca (pastoreo y suplemento con concentrado artesanal). A cada uno de los grupos se asignó uno de los siguientes tratamientos: T1 como grupo control sin suplementación adicional; T2 con un suplemento yodo estable suministrado por dosificación parenteral intramuscular en dosis única de $3 \mathrm{ml} / 100 \mathrm{Kg}$ de peso vivo cada 15 días para un total de 10 aplicaciones durante el experimento (la cantidad biodisponible de yodo para T2 fue de $620,4 \mathrm{mg} / \mathrm{vaca}$ en cada aplicación); T3 con un suplemento polimineral conformado por calcio, fósforo, selenio, zinc y yodo, y adicionalmente se suministró un compuesto polivitamínico de vitaminas del complejo B: B1, B2, B3, B6, B12, cada uno de los suplementos fueron aplicados quincenalmente por vía parenteral intramuscular en dosis única de $1 \mathrm{ml} / 20 \mathrm{Kg}$ de peso vivo (la cantidad biodisponible de minerales y vitaminas suministradas para cada vaca por aplicación fue: Calcio 100 mg, Fósforo 2000 mg, cinc 400 mg, selenio $4,4 \mathrm{mg}$, yodo $400 \mathrm{mg}$, vitaminas B1 200 $\mathrm{mg}$, B2 $60 \mathrm{mg}$, B3 $2000 \mathrm{mg}$, B6 $100 \mathrm{mg}$, у B12 $2000 \mathrm{mg}$ ).

Quincenalmente, se realizaron colectas de sangre y orina iniciando 30 días antes del parto y hasta el día 105 posparto, en cada colecta se tomaron muestras de sangre por venopunción coccígea en tubos sin anticoagulante, las muestras de orina se obtuvieron por masaje perineal, y se realizó la aplicación de los productos asignados a cada tratamiento.

Las muestras de sangre fueron trasladadas al laboratorio de metabolismo animal de la universidad nacional de Colombia, sede Palmira donde se centrifugaron a 2500 rpm por 25 minutos para la obtención de suero sanguíneo que fue almacenado en alícuotas en un freezer vertical a $-20^{\circ} \mathrm{C}$ para su posterior análisis.

Las muestras de orina fueron usadas para determinar la presencia de cetonas por medio de 
análisis químico macroscópico in situ utilizando tiras reactivas de orina (Multisti ${ }^{\circledR} 10 S G$, Siemens), las cuales permiten detectar la concentración de Acetoacetato (AcAc) y clasificarla dentro de los siguientes rangos: negativo, trazas $(0,49 \mathrm{mmol} / \mathrm{L})$, bajo $(1,46$ $\mathrm{mmol} / \mathrm{L})$, moderado (3,92 $\mathrm{mmol} / \mathrm{L})$, alto $(7,84 \mathrm{mmol} / \mathrm{L})$ y muy alto $(15,68 \mathrm{mmol} / \mathrm{L})$. La especificidad de la prueba es baja y la sensibilidad se encuentra entre 0,49 y $0,98 \mathrm{mmol} / \mathrm{L}$.

Los sueros sanguíneos fueron utilizados para determinar la concentración de ácidos grasos no esterificados (NEFA) por el método Acil Coenzima A Sintetasa/Ascorbato Oxidasa y de beta-hidroxibutirato (BHB) por el método NAD+/ 3-hidroxibutirato deshidrogenasa, ambos procedimientos realizados a partir de reactivos comerciales (NEFA y RANBUT, Randox Laboratories Antrim, Ireland). La medición de estos metabolitos se realizó por pruebas enzimáticas colorimétricas utilizando el equipo Analizador de química semiautomático de lectura óptica $R A Y T O \Omega$ (Shenzhen, China). Dentro de cada prueba se analizó una muestra de control bovino nivel 2 (valores fisiológicos), el cual se introdujo cada 15 muestras en el experimento y se usó como control de calidad intraensayo.

Los datos fueron almacenados en una base de datos de Excel y posteriormente procesados en el paquete estadístico InfoStat, versión 2014 (DI RIENZO et al., 2014), por medio de un diseño de medidas repetidas en el tiempo (procedimiento Modelos lineales generales y Mixtos), donde los efectos fijos fueron los tratamientos, los periodos y la interacción, mientras que el animal representó el efecto aleatorio y la covariable fue la producción de leche estimada a la lactancia. Para determinar la mejor estructura de covarianza se utilizaron el menor criterio de información Akaike (AIC) y el criterio de información bayesiano (BIC). Se realizó análisis descriptivo exploratorio unidimensional y las comparaciones entre medias con diferencias estadísticas se obtuvieron con la prueba DGC (Di Rienzo, Guzmán y Casanoves). Además, se usó el coeficiente de Pearson para determinar correlaciones entre variables.

\section{Resultados}

Se encontraron diferencias significativas entre periodos para $\mathrm{BHB}$, evidenciando una menor concentración en el preparto (días - 30 y -15) y el mayor valor en el día 15 posparto (Figura 1A). La interacción grupo - periodo mostró diferencias en los días 0,15 , y 45 para T1 y el día 30 para T2 con los valores más altos. La concentración media para los grupos fue $1,03 \pm 0,37 \mathrm{mmol} / \mathrm{L}$ para $T 1$, $0,82 \pm 0,22 \mathrm{mmol} / \mathrm{L}$ para $\mathrm{T} 2$, y $0,78 \pm 0,17 \mathrm{mmol} / \mathrm{L}$ para T3 sin diferencias significativas entre ellos.

Los NEFA presentaron diferencias significativas entre periodos; evidenciando la concentración más alta al momento del parto, seguido de los días -15 (preparto) y 15 (posparto), en relación a

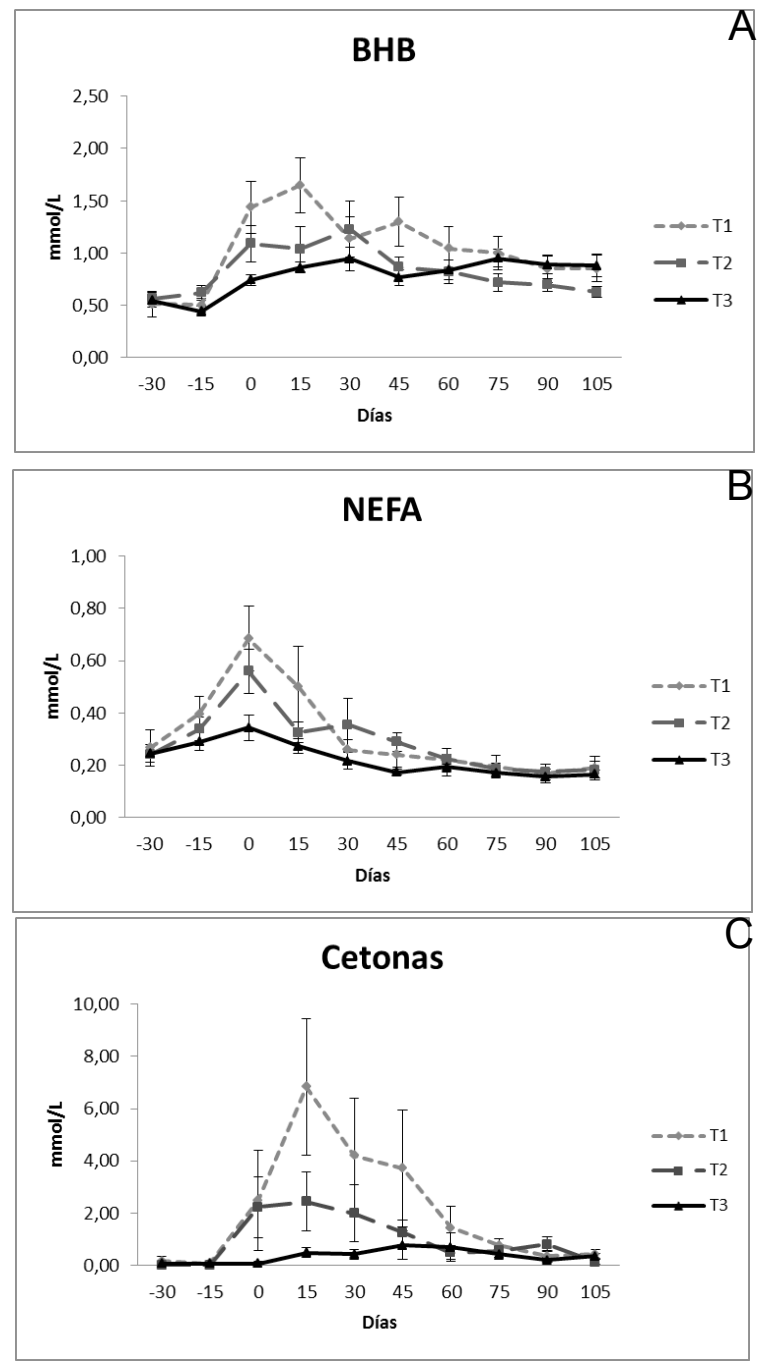

Figura 1. Comportamiento de los metabolitos energéticos para los tres grupos experimentales desde el día 30 antes del parto hasta el día 105 posparto, el día 0 corresponde al parto. Concentración sanguínea de A. BHB (mmol/L), B. NEFA (mmol/L), C. Cetonas $(\mathrm{mmol} / \mathrm{L})$. 
los demás periodos (Figura 1B). En la interacción grupo - periodo el valor más alto se halló en T1 para el día del parto. Los valores medios para cada uno de los grupos fueron de 0,31 $\pm 0,16 \mathrm{mmol} / \mathrm{L}$ para T1, 0,28 $\pm 0,11 \mathrm{mmol} / \mathrm{L}$ para T2 y $0,24 \pm 0,16$ $\mathrm{mmol} / \mathrm{L}$ para T3.

Para determinar la incidencia de cetosis subclínica en el experimento, se tuvo en cuenta el porcentaje de animales que por lo menos en uno de los periodos analizados exhibieron valores de $\mathrm{BHB}$ mayores a $1,00 \mathrm{mmol} / \mathrm{L}$, y para animales en riesgo de llegar a cetosis clínica se tuvo en cuenta el valor de $1,4 \mathrm{mmol} / \mathrm{L}$ (valores planteados por SUTHAR et al., 2013). La incidencia de cetosis subclínica fue de $71,4 \%$ en $\mathrm{T} 1,57,1 \%$ en $\mathrm{T} 2$ y $71,4 \%$ en T3, sin embargo, cuando se tuvieron en cuenta únicamente los animales que presentaron valores superiores a $1,4 \mathrm{mmol} / \mathrm{L}$, la incidencia de cetosis fue de $42,9 \%$ en T1, 28,6\% en T2 y $0 \%$ en T3.

Para el análisis de cetonas se utilizaron los valores medios asignados a la calificación cualitativa traza, baja, moderado, alta y muy alta, donde las concentraciones medias halladas en el experimento fueron $2,05 \pm 2,25 \mathrm{mmol} / \mathrm{L}$ para $\mathrm{T} 1$; $1,00 \pm 0,94 \mathrm{mmol} / \mathrm{L}$ para T2 y $0,36 \pm 0,25 \mathrm{mmol} / \mathrm{L}$ para T3. La concentración más alta de cetonas fue encontrada en T1 para el día 15 posparto (Figura $1 \mathrm{C}$ ) con un valor de $6,85 \mathrm{mmol} / \mathrm{L}$.

Las relaciones estadísticas entre la concentración de NEFA, BHB y cetonas se encuentran planteadas en la Tabla 2.

Tabla 2. Coeficiente de correlación de Pearson para las variables NEFA, BHB y Cetonas.

\begin{tabular}{cccc}
\hline & NEFA & BHB & Cetonas \\
\hline NEFA & 1,00 & & \\
BHB & $0,51<0,0001$ & 1,00 & \\
Cetonas & $0,51<0,0001$ & $0,72<0,0001$ & 1,00 \\
\hline
\end{tabular}

\section{Discusión}

Valores de $\mathrm{BHB}$ mayores a $1,00 \mathrm{mmol} / \mathrm{L}$ y hasta $1,40 \mathrm{mmol} / \mathrm{L}$ indican que los animales presentan cetosis subclínica; sin embargo, es común encontrar estos valores entre la primera y la tercera semana posparto como consecuencia de la alta movilización de lípidos que se presenta para suplir los requerimientos del inicio de la producción de leche. Por otra parte, concentraciones de BHB mayores a $1,40 \mathrm{mmol} / \mathrm{L}$ en la mayoría de casos están acompañadas de síntomas clínicos de cetosis (SUTHAR et al., 2013). En el presente trabajo se encontró que T1 mostró mayor tendencia a valores altos asociados con mayor riesgo de cetosis. Se ha encontrado que concentraciones de BHB mayores a 1,00 $\mathrm{mmol} / \mathrm{L}$ están asociadas con una menor tasa de preñez, y que la probabilidad de desarrollar desplazamiento de abomaso, cetosis clínica y metritis aumentan de 2,6 a 8 , de 3 a 6 y de 1 a 5,8 veces, respectivamente, y se agrava cuando los valores de BHB superan $1,20 \mathrm{mmol} / \mathrm{L}$ en las primeras 2 semanas posparto (SUTHAR et al., 2013).

La concentración de NEFA en plasma es usada como una herramienta de diagnóstico para evaluar el grado de BEN en las vacas lecheras en el posparto temprano, ya que este metabolito es considerado un indicador de movilización de reservas lipídicas en el organismo (QUIROZ et al., 2009). Al final de la lactancia e inicio del periodo seco se encuentran valores de NEFA menores a $0,2 \mathrm{mmol} / \mathrm{L}$, dos semanas anteriores al parto se incrementan gradualmente para llegar a su punto máximo entre los días 0 y 10 posparto con valores alrededor de $0,75 \mathrm{mmol} / \mathrm{L}$ (CONTRERAS y SORDILLO, 2011). DÍAZ et al. (2011) plantean que valores de NEFA hasta $0,25 \mathrm{mmol} / \mathrm{L}$ indican una estabilización en el balance energético, mientras concentraciones superiores a $0,4 \mathrm{mmol} / \mathrm{L}$ exhiben una intensa lipomovilización, y mayores a 0,7 $\mathrm{mmol} / \mathrm{L}$ aumentan el riesgo de cetosis, además, CONTRERAS y SORDILLO (2011), plantean que es común encontrar una concentración alta de este metabolito en animales obesos o con condición corporal mayor o igual a 4 puntos en la escala de ganado lechero. En la presente investigación se encontraron concentraciones superiores a 0,4 $\mathrm{mmol} / \mathrm{L}$ para el día del parto en T1 y T2, y para el día 15 posparto para T1, lo que puede haber incidido en la presentación de cetosis subclínica.

Los cuerpos cetónicos son altamente volátiles, por tanto, no solo se pueden encontrar en sangre, sino que también son excretados en fluidos como leche y orina (KROGH et al., 2011), por tal razón se han desarrollado pruebas que permiten 
una identificación rápida de estos compuestos utilizando dichos fluidos. Una de las principales pruebas desarrolladas para evaluar y determinar la concentración de cuerpos cetónicos es la realizada en tiras reactivas de orina, la cual es una prueba semicuantitativa que mide la concentración de AcAc, y cuenta con especificidad y sensibilidad aceptable en comparación con la prueba diagnóstica de mayor precisión que es la medición de BHB en sangre (LEBLANC, 2010).

KROGH et al. (2011) plantea que la prueba en orina se considera positiva a cetosis cuando el valor que se obtiene de AcAc es igual o superior al nivel moderado $(3,92 \mathrm{mmol} / \mathrm{L})$, sin embargo CARRIER et al. (2004) y LEBLANC (2010) difieren de esta afirmación, determinando que animales con valores por encima del umbral bajo $(1,46 \mathrm{mmol} / \mathrm{L})$ deben ser considerados en riesgo de presentar cetosis y deben recibir tratamiento preventivo. Los resultados encontrados en el presente trabajo exhiben una concentración mayor al valor de umbral moderado para el día 15 después del parto en $\mathrm{T} 1$, mientras que superiores al umbral bajo se encontraron en los días 0,15 y 30 posparto para T1 $(2,49 \pm 5,08$ $\mathrm{mmol} / \mathrm{L}, 6,85 \pm 6,92 \mathrm{mmol} / \mathrm{L}$ y $4,20 \pm 5,80 \mathrm{mmol} / \mathrm{L}$ ) у T2 $(2,24 \pm 3,08 \mathrm{mmol} / \mathrm{L} ; 2,45 \pm 2,95 \mathrm{mmol} / \mathrm{L}$ y $2,00 \pm 2,88 \mathrm{mmol} / \mathrm{L})$. Los resultados hallados indican que todas las vacas que participaron en el experimento excretaron cuerpos cetónicos en orina, específicamente aceto-acetato, cuyo origen más probable es la movilización de tejido adiposo.

Las relaciones estadísticas encontradas en el coeficiente de correlación de Pearson (Tabla 2) evidenciaron alta asociación entre la prueba de cetonas en tiras reactivas para orina y los metabolitos sanguíneos analizados (BHB y NEFA). Lo cual indica que el uso de las tiras reactivas para orina podría ser aplicado como un método de detección rápida de cetosis subclínica, siendo una prueba económica que se puede realizar en campo. Los resultados encontrados en el presente experimento coinciden con los planteados por KROGH et al. (2011), donde se encontró una fuerte asociación de la prueba de tiras reactivas de orina con diferentes análisis realizados en leche, concluyendo que diferentes fluidos pueden ser usados en la determinación de cetonas y el diagnostico de cetosis.
NEFA, BHB y cetonas son metabolitos que permiten evaluar el grado de lipomovilización que se presenta en el organismo, los resultados encontrados se asemejan a los hallados en otros trabajos realizados en condiciones de trópico (CAMPOS et al., 2007), donde los metabolitos presentan un comportamiento característico durante el periodo de transición con aumento en NEFA, BHB y cetonas al momento del parto, lo cual puede ser atribuido a la disminución en el consumo de materia seca que se inicia tres semanas antes del parto (FENWICK et al., 2008) y el cual, debe recuperarse cuando la homeostasis lleva a que los metabolitos se estabilicen en el posparto temprano, aproximadamente entre la segunda y cuarta semana después del parto (ESPOSITO et al., 2014).

En relación al comportamiento metabólico general, a pesar de que entre los grupos no se presentaron diferencias estadísticas, en la gráfica 1 ( $A, B$ y $C$ ) se puede observar como los grupos suplementados (T2 y T3) evidenciaron un mejor comportamiento metabólico que el grupo control. Lo cual podría estar explicado en el aporte homeostático brindado por la composición mineral y vitamínica de los suplementos suministrados, los cuales son importantes moduladores en el metabolismo y participan en diferentes procesos del organismo y en la prevención de enfermedades características del posparto temprano en vacas de alto rendimiento.

En T2 se suministró un suplemento a base de yodo, donde el mejor comportamiento de este grupo en comparación con el grupo control frente a la movilización de lípidos, puede haberse dado porque el yodo es un mineral esencial que participa en la formación de las hormonas tiroideas (triyodotironina y tiroxina), las cuales a su vez, actúan en la regulación de diferentes procesos en el organismo, como son el metabolismo basal, la glucogénolisis, la gluconeogénesis, el metabolismo de lípidos, metabolismo de proteínas, y en la lactogénesis (NUDDA et al., 2013; SAMANC et al., 2010). Se considera que el aporte adicional de yodo pudo haber estimulado una mayor formación de hormonas tiroideas con lo que se favoreció la regulación fisiológica del animal en una etapa de diferentes cambios metabólicos y nutricionales. 
En T3, que fue el grupo que evidenció una menor lipomovilización se suministró fósforo y vitamina $\mathrm{B} 12$, los cuales han sido sugeridos como tratamiento preventivo de cetosis, ya que la vitamina B12 puede estimular el proceso de gluconeogénesis mediante el incremento en la actividad de la enzima metilmalonil-coenzima A mutasa, la cual participa activamente en el ciclo de Krebs, mientras que el fósforo también participa en varias etapas de la gluconeogénesis realizando el proceso de fosforilación de los compuestos intermediarios para que puedan ingresar al ciclo y finalmente producir la energía necesaria (GORDON, 2013). Además el fósforo participa en otras funciones biológicas como el equilibrio ácido-base, el mantenimiento celular y la generación de adenosin trifosfato (ATP). El calcio también presente en T3 participa en la excitabilidad muscular, la coagulación de la sangre, la secreción de hormonas a partir de las células donde se sintetizan, además una considerable cantidad de calcio es direccionada al calostro y la leche (GOFF, 2006). El selenio y el zinc han sido altamente reconocidos por su actuación como agentes antioxidantes en el organismo permitiendo reducir el estrés oxidativo que se presenta durante el periodo de transición, a la vez que participan en la conformación de sistemas enzimáticos de diversos procesos metabólicos (CALAMARI et al., 2011; SOBHANIRAD et al., 2010). Por otra parte las vitaminas del complejo $B$ son indispensables en los mamíferos, y aunque los rumiantes las obtienen por síntesis microbiana en el rumen, la alta especialización de estos animales, ha aumentado sus requerimientos al punto que no se pueden cubrir completamente desde el rumen y es necesario suplementarlas en el periodo de transición, estas vitaminas participan como cofactores en diferentes ciclos metabólicos como la gluconeogénesis y la regeneración de grupos metilo (JUCHEM et al., 2012), así como la vitamina B12 se destaca por su participación en la gluconeogénesis, la vitamina B3 adquiere alta importancia en la prevención de cetosis por su capacidad para detener la movilización de NEFA, al generar una modificación en la síntesis y excreción de lipoproteínas hepáticas (SACADURA et al., 2008; KASHFI et al., 2011).

\section{Conclusiones}

La incidencia de cetosis subclínica fue menor en los grupos suplementados, indicando que un mejor aporte de vitaminas y minerales puede favorecer el metabolismo global de las vacas de alta producción reduciendo la movilización de reservas corporales al apoyar el proceso de adaptación a un nuevo estado fisiológico.

El mayor aporte de yodo durante el periodo de transición pudo favorecer la formación de hormonas tiroideas, las cuales participan en diferentes procesos metabólicos y nutricionales, incluidos el metabolismo de lípidos y la regulación homeostática, con lo cual podría explicarse el mejor desempeño metabólico de los animales suplementados, en comparación con el grupo control.

La menor movilización de reservas corporales que se encontró en el tratamiento de suplementación mixta con vitaminas y minerales podría estar determinada por una mejor adaptación al periodo de transición, dada por un suministro estratégico de micronutrientes que favorecen la homeostasis del organismo, acelerando el retorno a la estabilidad metabólica de las vacas lecheras.

La correlación que se presentó entre la concentración de cetonas mediante la prueba de tiras reactivas de orina, con los metabolitos sanguíneos indicadores de movilización de reservas corporales, BHB y NEFA, permite sugerir que la detección de cetonas en orina es un forma de identificación rápida de cetosis subclínica de gran utilidad, por su bajo costo y facilidad de uso, lo cual es importante en pruebas diagnósticas a nivel de campo.

Agradecimientos: A "Lácteos el Hato" por permitir el desarrollo del experimento en su finca ganadera y su colaboración durante la fase experimental, y a la vicerrectoría de investigación de la Universidad Nacional de Colombia sede Palmira por la financiación del proyecto. 


\section{Referencias}

BERGE, A.; VERTENTEN, G. 2014. A field study to determine the prevalence, dairy herd management systems, and fresh cow clinical conditions associated with ketosis in western European dairy herds. Journal of Dairy Science 97 (4):2145-154.

CALAMARI, L.; PETRERA, F.; ABENI, F.; BERTIN, G. 2011. Metabolic and hematological profiles in heat stressed lactating dairy cows fed diets supplemented with different selenium sources and doses. Livestock Science 142 (3):128-137.

CAMPOS, R.; CUBILLOS, C.; RODAS, A. 2007. Indicadores metabólicos en razas lecheras especializadas en condiciones tropicales en Colombia. Acta Agronómica 56 (2):85-92.

CAMPOS, R.; GONZÁLEZ, F.; COLDEBELLA, A.; LACERDA, L. 2005. Determinação de corpos cetônicos na urina como ferramenta para o diagnóstico rápido de cetose subclínica bovina e relação com a composição do leite. Archives of Veterinary Science 10 (2):49-54.

CARRIER, J.; STEWART, S.; GODDEN, S.; FETROW, J.; RAPNICKI, P. 2004. Evaluation and use of three cowside test for detection of subclinical ketosis in early postpartum cows. Journal of Dairy Science 87 (11):3725-3735.

CONTRERAS, G.; SORDILLO, L.M. 2011. Lipid mobilization and inflammatory responses during the transition period of dairy cows. Comparative Immunology, Microbiology, and Infectious Diseases 34 (3):281-289.

DI RIENZO J.A.; CASANOVES F.; BALZARINI M.G.; GONZALEZ L.; TABLADA M.; ROBLEDO C.W. 2014. InfoStat. [programa del ordenador]. versión 2014. Grupo InfoStat, FCA, Universidad Nacional de Córdoba, Argentina. Disponible en: http://www.infostat.com.ar.

DÍAZ, F.; MUIÑO, R.; PEREIRA, V.; CAMPOS, R.; BENEDITO, J. 2011. Relationship among blood indicators of lipomobilization and hepatic function during early lactation in high-yielding dairy cows. Journal of Veterinary Science 12 (3):251-255.

DUQUE, M.; OLIVERA, M.; ROSERO, R. 2011. Metabolismo energético en vacas durante la lactancia temprana y el efecto de la suplementación con grasa protegida. Revista Colombiana de Ciencias Pecuarias 24:74-82.

ESPOSITO, G.; IRONS, P. C.; WEBB, E. C.; CHAPWANYA, A. 2014. Interactions between negative energy balance, metabolic diseases, uterine health and immune response in transition dairy cows. Animal Reproduction Science 144 (3-4):60-71.

FENWICK, M.; FITZPATRICK, R.; KENNY, D.; DISKIN, M.; PATTON, J., MURPHY, J., WATHES, C. 2008. Interrelationships between negative energy balance (NEB) and IGF regulation in liver of lactating dairy cows. Domestic Animal Endocrinology 34 (1):31-44.

GARRO, C.; MIAN, L.; COBOS, M. 2013. Subclinical ketosis in dairy cows: prevalence and risk factors in grazing production system. Journal of Animal Physiology and Animal Nutrition (Berl) 98 (5):838-844.

GOFF, J. 2006. Macromineral physiology and application to the feeding of the dairy cow for prevention of milk fever and other periparturiend mineral disorders. Animal Feed Science and Technology 126 (34):237-257. 
GORDON, L. 2013. Ketosis treatment in lactating dairy cattle. The Veterinary Clinics of North America Food Animal Practice 29 (2):433-445.

GROSS, J.; SCHWARZ, F. J.; EDER, K.; VAN DORLAND, H. A.; BRUCKMAIER, R. M. 2013. Liver fat content and lipid metabolism in dairy cows during early lactation and during a mid-lactation feed restriction. Journal of Dairy Science 96 (8):5008-5017.

HOLDRIDGE, L. 1987. Ecología basada en zonas de vida. Instituto Interamericano de Cooperación para la Agricultura (IICA). San José, Costa Rica.

JUCHEM, S.O.; ROBINSON, P.H.; EVANS, E. 2012. A fat based rumen protection technology postruminally delivers a $B$ vitamin complex to impact performance of multiparous Holstein cows. Animal Feed Science and Technology 174 (1-2):68-78.

KASHFI, H.; YAZDANI, A.R.; LATIFI, M.; BIDABADI, F. S. 2011. Economic and managerial analysis of effective managerial strategies on prevention from ketosis in transition period in shahroud commercial dairy farms. ISRN Veterinary Science, (2011):1-8.

KROGH, M.A.; TOFT, N.; ENEVOLDSEN, C. 2011. Latent class evaluation of a milk test, a urine test, and the fat-to-protein percentage ratio in milk to diagnose ketosis in dairy cows. Journal of Dairy Science 94 (5):2360 - 2367.

LEBLANC, S. 2010. Monitoring metabolic health of dairy cattle in the transition period. Journal of reproduction and Development 56 (2010):S29-35.

NORO, M.; BARBOZA, C. 2012. Cetosis en rebaños lecheros: presentación y control. Spei Domus 8 (17):1-11.

NUDDA, A.; BATTACONE, G.; BOMBOI, G.; FLORIS, B.; DECANDIA, M.; PULINA, G. 2013. Effect of dietary iodine on thyroid hormonas and energy blood metabolites in lactating goats. Animal 7 (1):60-65.

QUIROZ, G.; LEBLANC, S.; DUFFIELD, T.; WOOD, D.; LESLIE, K.; y JACOBS, R. 2009. Reference limits for biochemical and hematological analytes of dairy cows one week before and one week after parturition. Canadian Veterinary Journal 50 (4):383-388.

SACADURA, F.C.; ROBINSON, P.H.; EVANS, E.; LORDELO, M. 2008. Effects of a ruminally protected B-vitamin supplement on milk yield and composition of lactating dairy cows. Animal Feed Science and Technology 14 (4):111-124

SAMANC, H.; STOJIC, V.; KIROVSKI, D.; JOVANOVIC, M.; CERNESCU, H.; VUJANAC, I. 2010. Thyroid hormones concentrations during the mid-dry period: An early indicator of fatty liver in Holstein-Friesian dairy cows. Journal of Thyroid Research 2010 (2010):1-6.

SOBHANIRAD, S.; CARLSON, D.; BAHARI, R. 2010. Effect of zinc methionine or zinc sulfate supplementation on milk production and composition of milk in lactating dairy cows. Biological Trace Element Research 136 (1):48-54.

SUTHAR, V.S.; CANELAS-RAPOSO, J.; DENIZ, A.; HEUWIESER, W. 2013. Prevalence of subclinical ketosis and relationships with postpartum diseases in European dairy cows. Journal of Dairy Science 96 (5):2925-2938. 
WEBER, C.; HAMETNER, C.; TUCHSCHERER, A.; LOSAND, B.; KANITZ, E.; OTTEN, W.; SAUERWEIN, R.M.; BECKER, F.; KANITZ, W.; y HAMMON, H.M. 2013. Hepatic gene expression involved in glucose and lipid metabolism in transition cows: Effects of fat mobilization during early lactation in relation to milk performance and metabolic changes. Journal of Dairy Science 96 (9):5670-5681.

WILDE, D. 2006. Influence of macro and microminerals in the periparturient period on fertility in dairy cattle. Animal Reproduction Science 96 (3-4):240-249. 